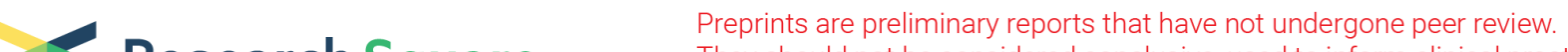 Research Square
or referenced by the media as validated information.
}

\section{Validation of the Relationship Between Coagulopathy and Localization of Hydroxyethyl Starch on the Vascular Endothelium in a Rat Hemodilution Model}

\section{Ryu Azumaguchi}

Sapporo Medical University: Sapporo lka Daigaku

Yasuyuki Tokinaga

Sapporo Medical University: Sapporo Ika Daigaku

Satoshi Kazuma ( $\triangle$ sea_hawk_3104@yahoo.co.jp)

Sapporo Medical University: Sapporo lka Daigaku https://orcid.org/0000-0002-7101-4202

Motonobu Kimizuka

Sapporo Medical University: Sapporo Ika Daigaku

Kosuke Hamada

Sapporo Medical University: Sapporo Ika Daigaku

Tomoe Sato

Sapporo Medical University: Sapporo Ika Daigaku

Michiaki Yamakage

Sapporo Medical University: Sapporo Ika Daigaku

\section{Research}

Keywords: Hydroxyethyl starch, von Willebrand factor, Coagulopathy, Glycocalyx, Localization, Endothelium, Shear viscosity

Posted Date: January 7th, 2021

DOl: https://doi.org/10.21203/rs.3.rs-138542/v1

License: (9) This work is licensed under a Creative Commons Attribution 4.0 International License. Read Full License 


\section{Abstract}

\section{Background}

Various anticoagulant properties have been associated with hydroxyethyl starch (HES), including coagulation disorders measured by point-of-care devices, decreases in von Willebrand factor and coagulation factor VIII, and inhibition of the interactions between platelet-membrane glycoproteins and of coagulation factors by coating of platelets. However, the mechanism for these properties remains unclear. The aim of this study was to test the hypothesis that coagulopathy induced by HES is caused by endothelial or glycocalyx damage as a result of localization of HES on the endothelium, due to the high shear viscosity of blood, using a rat model.

\section{Methods}

We compared blood coagulability measured by Sonoclot ${ }^{\circledR}$, levels of endothelial and glycocalyx damage markers and coagulation factors, and blood shear viscosity when hemodilution was performed with physiological saline (PS), 6\% HES 130/0.4 in PS (HES130) and 10\% HES 200/0.5 in PS (HES200). To precisely evaluate the basic mechanism of coagulopathy induced by HES, we performed hemodilution with an emphasis on dilutional equality by taking into consideration the intravascular residual rates of the infusion preparations. We also evaluated the localization rates of fluorescently labeled HES on endothelium in the isolated aorta. Statistical analyses were performed by one-way ANOVA followed by Tukey's test or Kruskal-Wallis test, and then Dunn's test for multiple comparisons.

\section{Results}

Sonoclot ${ }^{\circledR}$ measurements revealed that HES130 and HES200 decreased the fibrin gel formation rate to a greater extent than PS, and HES200 decreased the rate more than HES130. Similarly, HES130 and HES200 decreased von Willebrand factor levels to a greater extent than PS. HES130 and HES200 had a more protective effect than PS, with no evidence of damage to the endothelium or glycocalyx. Shear viscosity was variable between all pairs, and was lowest for PS and highest for HES200. HES130 and HES200 demonstrated comparable degrees of localization on the endothelium.

\section{Conclusions}

HES was shown to cover the endothelium, possibly due to its high shear viscosity, and this mechanism potentially acted to protect the endothelium and glycocalyx. However, this covering effect may be the cause of coagulopathy due to inhibition of von Willebrand factor secretion from the endothelium.

\section{Background}

Hydroxyethyl starch (HES) is believed to have a greater inhibitory effect on blood coagulation than physiological saline (PS) [1]. It has been reported that in vivo hemodilution with HES resulted in increased perioperative blood loss [2], and that in vitro or in vivo hemodilution caused coagulopathy measured by 
point-of-care devices such as ROTEM ${ }^{\circledR}, T E G \AA$, and Sonoclot ${ }^{\circledR}[3]$ as well as reductions in von Willebrand factor (VWF) antigen (Ag) levels and coagulation factor VIII (FVIII) Ag levels [4]. It has also been reported that the coating of platelets with HES molecules may inhibit contact of glycoprotein (GP) Ilb/Illa or GP Ib and coagulation factors on the platelet membrane [5]. However, the underlying mechanism integrating these phenomena is unknown.

The vascular endothelium (ET) and glycocalyx (GCX) are known to play a role in controlling blood coagulation by retaining thrombomodulin (TM) and heparan sulfate proteoglycan (HSPG) and by secreting VWF [6]. Recent studies on sepsis and trauma have focused on damage to the ET and GCX as a mechanism for causing blood coagulation disorders [7, 8]. Septic and traumatic inflammatory reaction are known to damage and elute TM on the ET, and HSPG in the GCX. Eluted TM in the blood activates protein C (PC), and activated PC (aPC) neutralizes coagulation factors V (FV) and FVIII. Eluted HSPG acts as an auto-anticoagulant substance. Recent studies have shown that HES is more damaging to the endothelium than albumin, and crystalloid is more damaging than HES $[9,10]$. However, a subgroup analysis in a clinical trial reported that HES produced a greater reduction in coagulability compared with albumin by ROTEM ${ }^{\circledR}$, yet the mechanism was shown to be unrelated to ET or GCX damage [11].

In a classic animal study, radiocarbon-labeled dextran molecules were shown to be 'adhered' to the vascular ET via a non-covalent bond, which led to the supposition that dextran was involved in suppressing the process of hemostasis by blocking contact between coagulation factors and the ET [12]. However, the precise mechanism explaining the action of HES on the ET and GCX is currently unknown. Furthermore, the relationship between the anticoagulant effects of HES and damage to the ET and GCX has also not been established.

Therefore, in an attempt to clarify the mechanisms underlying the anticoagulant effects of HES, we hypothesized that HES causes coagulopathy by localization and impairment of the ET and GCX, and that its high shear viscosity due to non-covalent binding is the cause of HES localization because of the inherently immobilizing nature of high viscosity material.

\section{Methods}

\section{Study design}

The present study was an animal experimental study using a rat hemodilution model.

\section{Study animals}

The Animal Research Ethics Committee of our university granted approval for the study (Permit Number: 16-095). All animal experiments were conducted during 2017-2019 in accordance with the ARRIVE guidelines. Male Wistar rats weighing 280-320 g were purchased from Japan SLC (Shizuoka, Japan) and kept in temperature $\left(22^{\circ} \mathrm{C}-24^{\circ} \mathrm{C}\right)$ and humidity $(45 \%-55 \%)$ controlled rooms with a 24-hour light-dark 
cycle, and given standard chow and tap water ad libitum. All surgical procedures were performed under anesthesia, and every effort was made to minimize suffering.

\section{Determination of sample size}

Sample size was determined using G*Power version 3.1.9.2. for analysis of the preliminary data for experimental hemodilution in which Sonoclot ${ }^{\circledR}$ was used, and focused on measurement of the clot rate (CR). The level of statistical significance was set at $5 \%$, the power was set at $80 \%$ and the number of groups was set at four. The calculated effect size was 0.57 , and the total sample size needed for each experiment was calculated to be 40 .

\section{Allocation of experimental animals}

Forty rats were divided into four groups of ten rats in each group. One group was assigned to the beforedilution group (control) and three hemodilution groups were assigned as follows: 1) PS, 2) $6 \% \mathrm{HES}$ 130/0.4 in PS (6\% Voluven; Fresenius Kabi AG, Bad Homburg, Germany) (HES130), and 3) 10\% HES 200/0.5 in PS (10\% Pentaspan; Jeil Pharm., Seoul, Korea) (HES200).

\section{Experimental protocol for preliminary hemodilution}

Rats were tracheostomized under inhalational anesthesia with $5 \%$ sevoflurane and $1 \mathrm{~L} / \mathrm{min} 100 \%$ oxygen during spontaneous breathing. A venous catheter was inserted percutaneously in the tail vein and an arterial catheter was inserted in the femoral artery by the open approach under microscopic visualization. Subsequent procedures were performed under mechanical ventilation ( $2 \%$ sevoflurane) with continuous blood pressure monitoring. Body temperature was kept at $37^{\circ} \mathrm{C}$ using an experimental animal heater (BWT-100A; Bio Research Center, Nagoya, Japan).

Each infusion preparation was administered at $1 \mathrm{ml} / \mathrm{hr}$ in the vein and $3 \mathrm{ml} / \mathrm{hr}$ in the artery. Anticoagulant agents were not used in order to exclude their potential effects on coagulability, blood viscosity, and ET and GCX markers. Intra-catheter blood clotting was avoided by maintaining arterial infusion at the minimum rate. Arterial blood sampling was performed every 20 minutes and included $0.2 \mathrm{ml}$ samples for blood gas analysis (EPOC; Epocal, Ottawa, Ontario, Canada) and $0.8 \mathrm{ml}$ samples for hemodilutional blood removal. Blood aspiration was performed gently, to avoid unnecessary platelet activation. Each blood sampling and blood removal was followed by a $1 \mathrm{ml}$ bolus infusion of the respective infusion preparation for volume compensation and arterial catheter flushing. An additional $0.5 \mathrm{ml}$ intravenous administration for each infusion preparation was permitted without limitation to maintain a stable blood pressure and to avoid decreases of more than $20 \%$ in mean blood pressure. These infusion and aspiration procedures resulted in gradual in vivo hemodilution without clinical evidence of shock.

Serial hemodilution was initiated according to the procedures described above until the hematocrit $(\mathrm{Ht})$ level was reduced to $31 \%$ or less (Figure 1). These procedures were defined as preliminary hemodilution. Fluid balance per body weight and the time required to achieve preliminary hemodilution were recorded. 
Following the preliminary hemodilution, hemodilution was continued for coagulability measurement, quantification of ET and GCX damage markers and coagulation factors, and shear viscosity measurement.

\section{Coagulability measurement using Sonoclot ${ }^{\circledR}$}

Sonoclot ${ }^{\circledR}$ (Sonoclot II Coagulation and Platelet Function Analyzer; Sienco, Morrison, CO, USA) is a pointof-care device for evaluation of whole blood clotting ability. The required blood sampling volume is $0.4 \mathrm{ml}$ per channel, with a total of $0.8 \mathrm{ml}$ for the two channels in the device. A vertically vibrating probe with a frequency of $200 \mathrm{~Hz}$ detects whole blood coagulation resistance that is output as a resistance curve, after activation by celite prefilled in a disposable cuvette. Activated clotting time (ACT) was defined as the time to start of blood clotting and represents the length of the horizontal part of the curve prior to the upslope of coagulation resistance. CR was defined as the rate of fibrin gel formation from fibrinogen, and represents the upslope angle of the coagulation resistance curve. Platelet function (PF) was defined as the strength of clot retraction. Sonoclot ${ }^{\circledR}$ was selected for use based on previous studies that demonstrated its effective detection of coagulopathy induced by hemodilution with crystalloids and colloids in vitro [13] and in vivo [14].

\section{Quantification of ET and GCX damage markers and coagulation factors by ELISA}

Commercially available ELISA kits were used for quantification. TM Ag (E-EL-R0960; Elabscience Biotechnology, Wuhan, China) and aPC Ag (SEA738Ra; Cloud-Clone, Katy, TX) were assayed to evaluate the extent of vascular ET damage by HES. Syndecan-1 (SDC-1) Ag (E-EL-R0996; Elabscience Biotechnology), which is known as a component of GCX and elutes into the blood when GCX is impaired, and HSPG Ag (E-EL-R0491; Elabscience Biotechnology) were assayed to evaluate the extent of GCX damage by HES. VWF Ag (E-EL-R1079; Elabscience Biotechnology), FV Ag (E-EL-R0230; Elabscience Biotechnology) and FVIII Ag (MBS749830; MyBioSource, San Diego, CA) from plasma were quantified to investigate the influence of these coagulation factors on the CR measured by Sonoclot ${ }^{\circledR}$. The whole blood volume required for all ELISA tests was calculated to be $5 \mathrm{ml}$, including storage, for each rat.

\section{Shear viscosity measurement using a rheometer}

The shear viscosity of whole blood was measured using a rheometer (MCR102, CP50-1; Anton Paar, Graz, Austria). Recordings were performed when the shear rate ranged from 1 to $1000 \mathrm{~s}^{-1}$ at a temperature of $37^{\circ} \mathrm{C}$. The shear viscosity at a shear rate of $398 \mathrm{~s}^{-1}$ was adopted and compared between the beforedilution group and each of the three dilution groups. This shear rate has previously been estimated to be close to that of the rat femoral artery and descending aorta [15]. Each $1.3 \mathrm{ml}$ blood sample obtained was added to a collecting tube prefilled with ethylenediaminetetraacetic acid to prevent clotting, and all of it was used for the measurement.

Secondary hemodilution and sampling protocol following preliminary hemodilution 
Following preliminary hemodilution, secondary hemodilution was continued in a different way for each of the three measurements until the $\mathrm{Ht}$ level was reduced to the range of $26 \%-30 \%$ (Figure 1 ). This hemodilution target was set prospectively because it is considered to approximate the conditions commonly found in clinical practice. All results were collected when the Ht levels were in the range of $26 \%-30 \%$ except in the before-dilution group. To minimize invasiveness, blood samples for measurements and quantifications in the before-dilution group were collected just after arterial cannulation, without tracheostomy.

Coagulability measurements after hemodilution in which the $\mathrm{Ht}$ level was $31 \%$ or less were performed using $0.2 \mathrm{ml}$ blood samples for blood gas analysis, $0.8 \mathrm{ml}$ blood samples for Sonoclot ${ }^{\circledR}$ measurement, and $1 \mathrm{ml}$ arterial bolus infusion every 20 minutes during a $1 \mathrm{ml} / \mathrm{hr}$ venous infusion and $3 \mathrm{ml} / \mathrm{hr}$ arterial infusion. Additional $0.5 \mathrm{ml}$ infusions were performed a few times. The blood samples for blood gas analysis and Sonoclot ${ }^{\circledR}$ measurement were collected simultaneously. One to three Sonoclot ${ }^{\circledR}$ measurements were recorded when the $\mathrm{Ht}$ level was $26 \%-30 \%$. Values of $\mathrm{CR}$, ACT and PF were averaged in each rat for comparison between the before-dilution group and each of the three hemodilution groups.

Quantification of ET and GCX damage markers and coagulation factors and the shear viscosity measurements were performed continuously by the preliminary hemodilution protocol. Blood samples for these measurements and quantifications were collected at the time of the second $0.2 \mathrm{ml}$ blood gas analysis rather than at the $0.8 \mathrm{ml}$ removal because the $\mathrm{Ht}$ levels were expected to be in the target range of $26 \%-30 \%$ at this time. These blood samples were collected only once in each rat because the amount of blood required for these examinations was too large to be drawn more than twice. All blood samplings were matched in the range of $26 \%-30 \% \mathrm{Ht}$ using this protocol.

\section{Quantitative optical evaluation of fluorescently labeled HES in the isolated aorta}

Forty rats were divided into four groups of 10 rats in each group. Fluorescein isothiocyanate (FITC) labeled HES130 (FITC-HES130) was infused in the isolated aorta of one group; FITC-HES200 was infused in one group; and in two control groups, fluorescein sodium salt (uranine; 213-00092, FUJIFILM Wako Pure Chemical, Osaka, Japan) and either HES130 or HES200 was infused.

Rats were anesthetized by inhalation of $5 \%$ sevoflurane and were sacrificed under narcosis by common carotid artery resection. After thoracotomy, the descending aorta was isolated immediately and bathed in $37^{\circ} \mathrm{C}$ phosphate buffered saline (PBS). The aorta was prepared in $12 \mathrm{~mm}$ sections under microscopic visualization. An 18-gauge venous catheter (Supercath 5, Medikit, Tokyo, Japan) was cut in the midportion. The hub-side was inserted at the proximal end of the aorta and the tip was inserted at the distal end. Both sides were ligated with 3-0 silk thread. The amount of pigment contained in all infusion preparations was adjusted to $6.0 \times 10^{-5} \mathrm{~mol} / \mathrm{L}$. Infusion into the isolated aorta was performed with care to prevent the aorta from being unnecessarily bent or pulled. Each infusion preparation was infused at 5 $\mathrm{ml} / \mathrm{hr}$ for 20 minutes. For flushing, PBS was infused at $5 \mathrm{ml} / \mathrm{hr}$ for 20 minutes followed by a $5 \mathrm{ml}$ bolus infusion for 25 seconds. 
After infusion, all aortas were immediately frozen and sectioned on a cryostat (CM3050 S; Leica, Nussloch, Germany) at a thickness of $40 \mu \mathrm{m}$. Three cross-sections were collected, at distances of 2, 6, and $10 \mathrm{~mm}$ from the proximal end of the aorta (Figure 2a), and each was placed on a slide glass in a random orientation. The cross-sections were evaluated by a confocal laser scanning microscope (LSM 510 META; ZEISS, Jena, Germany). One cross-section was divided into 6 parts at $60^{\circ}$ intervals from the $30^{\circ}$ position, with $0^{\circ}$ as the $120^{\prime}$ clock position. Images of the $0^{\circ}, 120^{\circ}$ and $240^{\circ}$ sections were taken (Figure 2b). Image J public domain software (NIH Image, Bethesda, MD, USA) was used for image analysis. In each image, a line was drawn normal to the point on the ET where the luminance intensity was the highest $(P)$, and the middle point of this line was set on the outer membrane (Q) (Figure 2c). On the normal line, the ratio of the ET luminance intensity was determined when the outer membrane was set as a reference $(P / Q)$, to offset subtle differences in image shooting conditions (Figure $2 d$ ). Nine ET luminance intensity ratios were calculated per aorta and these results were averaged. Luminance intensity was compared among the four groups.

\section{Statistical analysis}

Statistical analyses were performed using Graph Pad Prism 7 (GraphPad Software, San Diego, CA, USA). Data distribution was assessed using the Shapiro-Wilk test. Data are presented as the mean (standard deviation) for normally distributed data or as the median (interquartile range) for other data. Statistical comparisons of normally distributed data were performed by one-way ANOVA followed by Tukey's test for multiple comparisons. Statistical comparisons of other data were performed by Kruskal-Wallis test followed by Dunn's test for multiple comparisons. $P$ values $<0.05$ were considered to be statistically significant.

\section{Results}

\section{Hemodilution}

The fluid balance per body weight needed for common preparatory hemodilution was significantly lower in all HES groups compared with the PS group $(P<0.0001)$ (Table 1). There were no significant differences in the time needed for targeted dilution. Final Ht dilution levels were $27-28 \%$ in all hemodilution groups, and were significantly lower compared with before dilution for each of the three measurements of coagulability, coagulation factors and endothelial and glycocalyx damage markers, and blood viscosity $(P<0.0001)$ (Table 1$)$. 
Table 1

Performance of hemodilution.

\begin{tabular}{|c|c|c|c|c|c|}
\hline & $\begin{array}{l}\text { Before } \\
\text { dilution }\end{array}$ & PS & HES130 & HES200 & $P$ \\
\hline \multicolumn{6}{|l|}{ Preliminary hemodilution } \\
\hline $\mathrm{n}$ & 30 & 30 & 30 & 30 & \\
\hline Fluid balance per body weight $\left(\cdot 10^{-3} \mathrm{ml} / \mathrm{g}\right)$ & & $\begin{array}{l}35 \\
(13)\end{array}$ & $\begin{array}{l}13(3.1) \\
\S\end{array}$ & $\begin{array}{l}11(6.2) \\
\S\end{array}$ & $<.0001$ \\
\hline \multicolumn{2}{|l|}{ Time needed for targeted dilution (min) } & $\begin{array}{l}102 \\
(28)\end{array}$ & $88(32)$ & $84(29)$ & 0.4 \\
\hline \multicolumn{6}{|l|}{$\begin{array}{l}\text { Hemodilution for the coagulability } \\
\text { measurement }\end{array}$} \\
\hline $\mathrm{n}$ & 10 & 10 & 10 & 10 & \\
\hline $\mathrm{Ht}(\%)$ & $\begin{array}{l}37 \\
(1.4)\end{array}$ & $\begin{array}{l}28 \\
(0.93) \\
\star\end{array}$ & 28 (0.81)* & $28(1.1)$ & $<.0001$ \\
\hline \multicolumn{6}{|l|}{$\begin{array}{l}\text { Hemodilution for quantification of } \\
\text { endothelial and glycocalyx damage } \\
\text { markers and coagulation factors }\end{array}$} \\
\hline $\mathrm{n}$ & 10 & 10 & 10 & 10 & \\
\hline $\mathrm{Ht}(\%)$ & $\begin{array}{l}36 \\
(1.4)\end{array}$ & $\begin{array}{l}29 \\
(0.95)\end{array}$ & $\underset{*}{28}(1.1)$ & $28(1.1)$ & $<.0001$ \\
\hline \multicolumn{6}{|l|}{$\begin{array}{l}\text { Hemodilution for the shear viscosity } \\
\text { measurement }\end{array}$} \\
\hline $\mathrm{n}$ & 10 & 10 & 10 & 10 & \\
\hline $\mathrm{Ht}(\%)$ & $\begin{array}{l}38 \\
(1.7)\end{array}$ & $\begin{array}{l}28 \\
(1.4)\end{array}$ & $\begin{array}{l}28 \\
(0.88) *\end{array}$ & $27(1.1)$ & $\dot{0} 0.0001$ \\
\hline $\begin{array}{l}\text { Data are presented as the mean (SD). Ht, her } \\
\text { hydroxyethyl starch } 130 / 0.4 \text { in PS ( } 6 \% \text { Voluv } \\
\text { (10\% Pentaspan). }{ }^{*}<<0.0001 \text { vs before dilut }\end{array}$ & $\begin{array}{l}\text { rit; PS, phy } \\
\text { IES 200, } 10 \\
P<0.0001\end{array}$ & $\begin{array}{l}\text { iologice } \\
\% \text { hydro } \\
\text { s PS }\end{array}$ & $\begin{array}{l}\text { saline; } \mathrm{HES} \\
\text { ethyl starc }\end{array}$ & $\begin{array}{c}130,6 \% \\
200 / 0.5 \mathrm{i}\end{array}$ & PS \\
\hline
\end{tabular}

\section{Quantification Of Et And Gcx Damage Markers By Elisa}

TM Ag levels for the HES130 group (450 [250] pg/ml) and HES200 group (490 [270] pg/ml) were significantly lower compared with the before-dilution group $(1000[310] \mathrm{pg} / \mathrm{ml})(P=0.008, P=0.016$, respectively) and PS group (1100 [580] pg/ml) $(P=0.003, P=0.005$, respectively) (Fig. 4a). APC Ag levels for the HES200 group (11 [1.5] ng/ml) were significantly higher compared with the before-dilution group 
$(13[1.9] \mathrm{ng} / \mathrm{ml})(P=0.021)$. No significant difference was found among any other pairs (Fig. 4b). SDC-1 showed a very similar trend to TM (Fig. 4c). There was no significant difference in HSPG Ag levels among the groups $(P=0.98)$ (Fig. 4d).

\section{Quantification Of Coagulation Factors By Elisa}

VWF Ag levels for the HES130 and HES200 groups (each 10 [1.4] ng/ml) were significantly lower compared with the before-dilution group $(13[1.4] \mathrm{ng} / \mathrm{ml})(P=0.003)$ and $P S$ group $(13[2.1] \mathrm{ng} / \mathrm{ml})(P=$ 0.001) (Fig. 5a). FV Ag levels were significantly higher in the HES200 group $(25[19,32] \mathrm{ng} / \mathrm{ml})$ compared with the before-dilution group $(11[8.6,15] \mathrm{ng} / \mathrm{ml})(P=0.03)$. No significant difference was found among any other pairs (Fig. 5b). FVIII Ag levels for the PS group (72 [12] ng/ml) and the HES130 group (92 [13] $\mathrm{ng} / \mathrm{ml})$ were significantly lower compared with the before-dilution group $(120[21] \mathrm{ng} / \mathrm{ml})(\mathrm{P}<0.001, \mathrm{P}=$ 0.004 , respectively) and the HES200 group $(140[37] \mathrm{ng} / \mathrm{ml})(P<0.001, P=0.002$, respectively) (Fig. $5 \mathrm{c})$.

\section{Shear Viscosity Measurement By Rheometer}

Shear viscosity of blood was significantly different among all the hemodilution groups. Values of shear viscosity in the before-dilution, PS, HES130, and HES200 groups were 3.7 (0.17), 2.5 (0.13), 2.8 (0.13), and $3.0(0.16)(\mathrm{mPa} \cdot \mathrm{s})$, respectively (Fig. 6).

\section{Localization of fluorescently labeled HES in the vascular ET of the isolated aorta}

Cross-sectional images show localization of FITC-HES130 and FITC-HES200 on the vascular ET (Fig. 7a). The light intensity ratio at the outer membrane of the ET was significantly higher in both the FITC-HES130 group (9.0 [2.7]) and FITC-HES200 group (10.5 [2.2]) compared with fluorescein sodium salt in the HES130 group (1.5 [0.25]) $(P<0.0001)$ and fluorescein sodium salt in the HES200 group $(1.4[0.15])(P<$ $0.0001)$, respectively. There was no significant difference in light intensity ratio between the FITC-HES130 and FITC-HES200 groups $(P=0.49)$ (Fig. 7b).

\section{Discussion}

This study compared blood coagulability, levels of ET and GCX damage markers and coagulation factors, and shear viscosity under conditions of hemodilution performed equally with PS, HES130 and HES200. Additionally, vascular ET localization of fluorescently labeled HES130 and HES200 was examined. The results showed that HES impaired coagulability, yet acted protectively on the ET and GCX. HES resulted in increased shear viscosity and demonstrated localization to the vascular ET. To specifically evaluate the basic mechanisms of coagulopathy induced by HES, we chose the following constructs for evaluation: 1) use of an in vivo model, 2) standardization of factors affecting coagulation (e.g., surgical invasion), and 3) dilutional equality taking into consideration the intravascular residual rates of the infusion preparations. These conditions were chosen because it was necessary to create a hemodilution model 
that maintained cell metabolism by a-amylase, avoided unnecessary activation of coagulation factors or platelets, and essentially eliminated the dilutional effect itself. Our hemodilution model met all of these conditions. Hemodilution with HES increased the shear viscosity compared with PS, and HES200 significantly increased shear viscosity compared with HES130 (Fig. 6). HES was localized prominently on the ET (Fig. 7a, b). Although our study did not show a significant difference between HES130 and HES200, HES200 tended to be localized to a greater extent than did HES130 (Fig. 7b). Previous studies have demonstrated coating of HES around platelets [5] and localization of dextran on the ET [12], which suggests that artificial colloids tend to 'stick' to objects.

Contrary to our hypothesis, HES acted protectively on the ET and GCX compared with PS (Fig. 4a, c). The localizing and protective effects of HES on the ET were observed in the same manner with HES acting to a greater extent to protect the ET and GCX compared with PS (Fig. 7b). This result suggests that localization of HES on the ET and GCX may play a role in covering and protecting the ET and GCX. GCX functions primarily to regulate vascular permeability, and protection of GCX function would help to prevent leakage of the infused fluid [16]. This is consistent with the present result that the amount of infusion preparation used in the HES130 and HES200 groups was less than in the PS group (Table 1). In addition, previous studies have shown that volume overload causes ET and GCX damage by producing increases in atrial natriuretic peptide [17]. Likewise, increased fluid infusion has been correlated to GCX damage [18]. Therefore, the difference in the amount of infusion preparation itself could support the property that HES is protective to the ET and GCX. Ag levels of TM and SDC-1 in the PS group were not decreased by dilution (Fig. 4a, c). This result indicates that PS could potentially act to harm the ET and GCX and increase TM and SDC-1 Ag levels. It is unclear why there was no difference in levels of HSPG Ag, which is a marker of GCX damage as is SDC-1 (Fig. 4d). It can be presumed that the morphological difference between HSPG and SDC-1 [19] could be responsible for differences in fragility.

Interestingly, despite the protective action of HES on ET and GCX, HES impaired coagulability to a greater extent than did PS, as indicated by the decreased CR by Sonoclot ${ }^{\circledR}$ and the decreased VWF Ag levels (Fig. 3b, 5a). The CR by Sonoclot ${ }^{\circledR}$ mainly represents the degree of fibrin gel formation from fibrinogen. No studies have shown directly that a decrease in VWF Ag levels can be detected by Sonoclot ${ }^{\circledR}$. However,

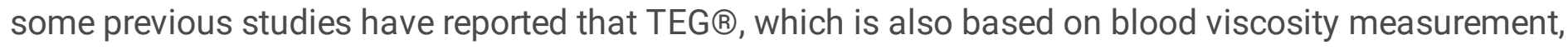
can detect a decrease in both Ag levels and activity of VWF [20]. Sonoclot ${ }^{\circledR}$ may also be capable of detecting decreases in VWF Ag levels because both tests monitor the process of whole blood clotting initiated by the addition of a coagulation activator. Decreases in VWF Ag levels showed a similar trend to the ET and GCX damage markers (Figs. 4a, c, 5a) and a negative correlation with the covering rate of HES on the ET (Fig. 7b). VWF is produced in ET cells and is mostly secreted directly, with some stored in the Weibel-Palade bodies, which are responsible for regulating acute secretion of VWF [21]. If there is copious acute VWF secretion and secretion points are limited, total VWF Ag levels decrease even when little of the ET surface is covered and the half-life of VWF is moderately long. To verify whether covering of the ET and GCX by HES for less than one hour can reduce VWF levels, it is necessary to confirm an increasing rate of VWF secretion in vivo during the acute phase. Significant decreases in VWF Ag levels or 
activity have not been observed in vitro [22], which also suggests that HES may inhibit VWF secretion from the ET.

PF measured by Sonoclot ${ }^{\circledR}$ was significantly high in both HES groups compared with the PS groups (Fig. 3c). This result is inconsistent with that of a previous study [5]. PF reflects strength of clot retraction in which the distance between platelets is shortened through the binding of Gllb/llla and fibrinogen or GPIIb/IIla and VWF during the blood clotting process [23]. A previous study reported that only HES130 did not decrease GPIIb/IIla on platelets, in contrast to the effects of HES200, HES450 and HES70 [24]. HES130 has been shown to activate immunological platelet function [25]. These findings agree with our results; however, the mechanism remains unknown. It is known that shear stress activates platelets [26] and increases as shear viscosity increases. Although viscosity was highest in the before-dilution group, there were no significant differences in PF values between the other groups (Fig. 3c). This finding can be explained by the argument that due to high shear viscosity, HES did not cause a decrease in PF values, which were predicted to decrease to the same extent as for PS by the dilution effect. This phenomenon may have only been observed to occur in cuvettes, where shear stress can be generated by vibration of the testing probe at $200 \mathrm{~Hz}$.

We found that aPC Ag levels were lower when using HES200 than before dilution (Fig. 4b). This finding is consistent with the decreased TM and increased FV Ag levels with HES200 compared with before dilution (Figs. 4a, 5b). Increased aPC Ag may be related to ET damage [10]. The decrease in aPC Ag could be explained by the dilutional effect and additional ET protection by HES200 in this situation.

FVIII Ag levels were lower for PS and HES130 compared with before dilution, but paradoxically were higher for HES200 compared with PS and HES130 (Fig. 5c). It is known that FVIII binds to VWF and remains stable in the bloodstream [27]. Even in patients with acquired von Willebrand disease, FVIII decreases as VWF Ag decreases [28]. Recent studies have reported spontaneous increase of VWF Ag levels in FVIII-deficient mice [29], decreased VWF Ag following administration of FVIII concentrate in patients with hemophilia [30], and decreased FVIII activity in vitro in the presence of VWF Ag [31]. These findings suggest that FVIII and VWF might be in a state of equilibrium, and the possibility that a decrease in VWF can produce a surplus of FVIII. Further research on the processing of FVIII and VWF is required. A prior study has reported that Sonoclot $\circledast$ was not able to detect increases in FVIII activity after administration of desmopressin [32]. The results of the present study indicate that the difference in FVIII Ag levels between HES200 and other infusion preparations may not have affected Sonoclot ${ }^{\circledR}$ measurement. In addition, decreased aPC Ag supported higher levels of FVIII Ag in HES200.

There were several limitations in our study. We were able to show that hemodilution with HES resulted in hemostatic impairment, ET and GCX protection, and localization of HES on the ET when the hemodilution was performed with HES; however, we did not demonstrate a direct interaction among these conditions. We quantified the Ag levels of coagulation factors but did not measure respective activity among them. FITC-HES was infused into the isolated aorta in a steady rather than pulsatile flow, and the infusion rate 
was slower than that of the real aorta. In addition, most of the infusion and blood sampling was arterial, which is different from actual clinical practice.

\section{Conclusions}

Our study showed that hemodilution with HES was associated with localization of HES on the ET and covering of the ET and GCX, which was probably due to the high shear viscosity of HES. HES may act to protect rather than damage the ET and GCX. Covering of the ET by HES can limit VWF secretion from the ET and cause coagulopathy by reducing VWF Ag levels. These findings may help to comprehensively explain the various anticoagulant effects of HES reported so far and to clarify the underlying mechanism of the anticoagulation and antiplatelet functions of HES.

\section{Abbreviations}

HES

hydroxyethyl starch

PS

physiological saline

$\mathrm{Ag}$

antigen

FVIII

coagulation factor VIII

GP

glycoprotein

ET

endothelium

GCX

glycocalyx

TM

thrombomodulin

HSPG

heparan sulfate proteoglycan

PC

protein $\mathrm{C}$

aPC

activated protein $\mathrm{C}$

FV

coagulation factor $\mathrm{V}$

CR

clot rate 
HES130

$6 \%$ HES 130/0.4 in PS

HES200

$10 \%$ HES 200/0.5 in PS

$\mathrm{Ht}$

hematocrit

ACT

activated clotting time

PF

platelet function

SDC-1

syndecan-1

FITC

fluorescein isothiocyanate

PBS

phosphate buffered saline

\section{Declarations}

\section{Ethics approval and consent to participate}

Approval was granted by the Animal Research Ethics Committee of our university (No: 16-095). All animal experiments were conducted in accordance with the ARRIVE guidelines.

\section{Consent for publication}

Not applicable

\section{Availability of data and materials}

The data collected and analyzed in the current study are available from the corresponding author on reasonable request.

\section{Competing interests}

The authors declare that they have no competing interests.

\section{Funding}

This study was partially supported by a Grant-in-Aid for Young Scientists (B) (No. 40737384) from the Japan Society for the Promotion of Science, Tokyo, Japan (to R.A.).

\section{Authors' contributions}


RA contributed to experimental protocol design, all phases of the research, and writing the manuscript. YT provided scientific interpretation and assisted with editing. SK, MK, KH and TS assisted with data collection. MY supervised the research. All authors read and approved the final manuscript.

\section{Acknowledgements}

The authors would like to thank Ms. Hiroko Kawakami and Dr. Taku Yoshiya (Peptide Institute Inc., 7-2-9 Saito-Asagi, Ibaraki-shi, Osaka, Japan) for preparing and providing the FITC-labeled HES powder.

\section{References}

1. Myburgh JA, Mythen MG. Resuscitation fluids. N Engl J Med. 2013;369:1243-51.

2. Navickis RJ, Haynes GR, Wilkes MM. Effect of hydroxyethyl starch on bleeding after cardiopulmonary bypass: a meta-analysis of randomized trials. J Thorac Cardiovasc Surg. 2012;144:223-30.

3. Hartog CS, Reuter D, Loesche W, Hofmann M, Reinhart K. Influence of hydroxyethyl starch (HES) $130 / 0.4$ on hemostasis as measured by viscoelastic device analysis: a systematic review. Intens Care Med. 2011;37:1725-37.

4. Levi M, Jonge Ed. Clinical relevance of the effects of plasma expanders on coagulation. Semin Thromb Hemost. 2007;33:810-5.

5. Deusch E, Gamsjäger T, Kress HG, Kozek-Langenecker SA. Binding of hydroxyethyl starch molecules to the platelet surface. Anesth Analg. 2003;97:680-3.

6. van Hinsbergh VW. Endothelium-role in regulation of coagulation and inflammation. Semin Immunopathol. 2012;34:93-106.

7. Johansson PI, Stensballe J, Ostrowski SR. Shock induced endotheliopathy (SHINE) in acute critical illness - a unifying pathophysiologic mechanism. Crit Care. 2017;21:25.

8. van Zyl N, Milford EM, Diab S, Dunster K, McGiffin P, Rayner SG, Staib A, Reade MC, Fraser JF. Activation of the protein $\mathrm{C}$ pathway and endothelial glycocalyx shedding is associated with coagulopathy in an ovine model of trauma and hemorrhage. J Trauma Acute Care Surg. 2016;81:674-84.

9. Torres LN, Chung KK, Salgado CL, Dubick MA, Torres Filho IP. Low-volume resuscitation with normal saline is associated with microvascular endothelial dysfunction after hemorrhage in rats, compared to colloids and balanced crystalloids. Crit Care. 2017;21:160.

10. Jacob M, Bruegger D, Rehm M, Welsch U, Conzen P, Becker BF. Contrasting effects of colloid and crystalloid resuscitation fluids on cardiac vascular permeability. Anesthesiology. 2006;104:1223-31.

11. Kammerer T, Hulde N, Speck E, Hübner M, Crispin A, Zwissler B, Conzen P, von Dossow V, Schäfer ST, Hofmann-Kiefer K, Rehm M. Effects of balanced hydroxyethyl starch $6 \%(130 / 0.4)$ and albumin $5 \%$ on clot formation and glycocalyx shedding: Subgroup analysis of a prospective randomized trial. Thromb Res. 2019;183:111-8. 
12. Bloom WL, Harmer DS, Bryant MF, Brewer SS. Coating of vascular surfaces and cells. A new concept in prevention of intravascular thrombosis. Proc Soc Exp Biol Med. 1964;115:384-6.

13. Casutt M, Kristoffy A, Schuepfer G, Spahn DR, Konrad C. Effects on coagulation of balanced (130/0.42) and non-balanced (130/0.4) hydroxyethyl starch or gelatin compared with balanced Ringer's solution: an in vitro study using two different viscoelastic coagulation tests ROTEM ${ }^{\text {TM }}$ and SONOCLOT ${ }^{\mathrm{TM}}$. Br J Anaesth. 2010;105:273-81.

14. Bischof DB, Ganter MT, Shore-Lesserson L, Hartnack S, Klaghofer R, Graves K, Genoni M, Hofer CK. Viscoelastic blood coagulation measurement with Sonoclot predicts postoperative bleeding in cardiac surgery after heparin reversal. J Cardiothorac Vasc Anesth. 2015;29:715-22.

15. Sakariassen KS, Orning L, Turitto VT. The impact of blood shear rate on arterial thrombus formation. Future Sci OA. 2015;1:FSO30.

16. Becker BF, Chappell D, Jacob M. Endothelial glycocalyx and coronary vascular permeability: the fringe benefit. Basic Res Cardiol. 2010;105:687-701.

17. Bruegger D, Jacob M, Rehm M, Loetsch M, Welsch U, Conzen P, Becker BF. Atrial natriuretic peptide induces shedding of endothelial glycocalyx in coronary vascular bed of guinea pig hearts. Am J Physiol Heart Circ Physiol. 2005;289:H1993-9.

18. Pouska J, Tegl V, Astapenko D, Cerny V, Lehmann C, Benes J. Impact of Intravenous Fluid Challenge Infusion Time on Macrocirculation and Endothelial Glycocalyx in Surgical and Critically III Patients. Biomed Res Int. 2018; 2018: 8925345.

19. Okada H, Takemura G, Suzuki K, Oda K, Takada C, Hotta Y, Miyazaki N, Tsujimoto A, Muraki I, Ando Y, Zaikokuji R, Matsumoto A, Kitagaki H, Tamaoki Y, Usui T, Doi T, Yoshida T, Yoshida S, Ushikoshi H, Toyoda I, Ogura S. Three-dimensional ultrastructure of capillary endothelial glycocalyx under normal and experimental endotoxemic conditions. Crit Care. 2017;21:261.

20. Regling K, Kakulavarapu S, Thomas R, Hollon W, Chitlur MB. Utility of thromboelastography for the diagnosis of von Willebrand disease. Pediatr Blood Cancer. 2019;66:e27714.

21. Giblin JP, Hewlett LJ, Hannah MJ. Basal secretion of von Willebrand factor from human endothelial cells. Blood. 2008;112:957-64.

22. von Roten I, Madjdpour C, Frascarolo P, Burmeister MA, Fisch A, Schramm S, Bombeli T, Spahn DR. Molar substitution and $\mathrm{C} 2 / \mathrm{C} 6$ ratio of hydroxyethyl starch: influence on blood coagulation. $\mathrm{Br} \mathrm{J}$ Anaesth. 2006;96:455-63.

23. Hett DA, Walker D, Pilkington SN, Smith DC. Sonoclot analysis. Br J Anaesth. 1995;75:771-6.

24. Franz A, Bräunlich P, Gamsjäger T, Felfernig M, Gustorff B, Kozek-Langenecker SA. The effects of hydroxyethyl starches of varying molecular weights on platelet function. Anesth Analg. 2001;92:1402-7.

25. Sossdorf M, Marx S, Schaarschmidt B, Otto GP, Claus RA, Reinhart K, Hartog CS, Lösche W. HES 130/0.4 impairs haemostasis and stimulates pro-inflammatory blood platelet function. Crit Care. 2009;13:R208. 
26. Gitz E, Koopman CD, Giannas A, Koekman CA, van den Heuvel DJ, Deckmyn H, Akkerman JW, Gerritsen HC, Urbanus RT. Platelet interaction with von Willebrand factor is enhanced by shearinduced clustering of glycoprotein Iba. Haematologica. 2013;98:1810-8.

27. Terraube V, O'Donnell JS, Jenkins PV. Factor VIII and von Willebrand factor interaction: biological, clinical and therapeutic importance. Haemophilia. 2010;16:3-13.

28. Lison S, Dietrich W, Spannagl M. Review article: unexpected bleeding in the operating room: the role of acquired von Willebrand disease. Anesth Analg. 2012;114:73-81.

29. Kiouptsi K, Grill A, Mann A, Döhrmann M, Lillich M, Jäckel S, Malinarich F, Formes H, Manukyan D, Subramaniam S, Khandagale A, Karwot C, Thal SC, Bosmann M, Scharrer I, Jurk K, Reinhardt C. Mice deficient in the anti-haemophilic coagulation factor VIII show increased von Willebrand factor plasma levels. PLoS One. 2017;12:e0183590.

30. van Bladel ER, Tuinenburg A, Roest M, de Groot PG, Schutgens RE. Factor VIII concentrate infusion in patients with haemophilia results in decreased von Willebrand factor and ADAMTS-13 activity. Haemophilia. 2014;20:92-8.

31. Butenas S, Parhami-Seren B, Mann KG. The influence of von Willebrand factor on factor VIII activity measurements. J Thromb Haemost. 2009;7:132-7.

32. Schött U. Sollén C, Axelsson K. Rugarn P, Allvin I. Desmopressin acetate does not reduce blood loss during total hip replacement in patients receiving dextran. Acta Anaesthesiol Scand. 1995; 39: 5928.

\section{Figures}




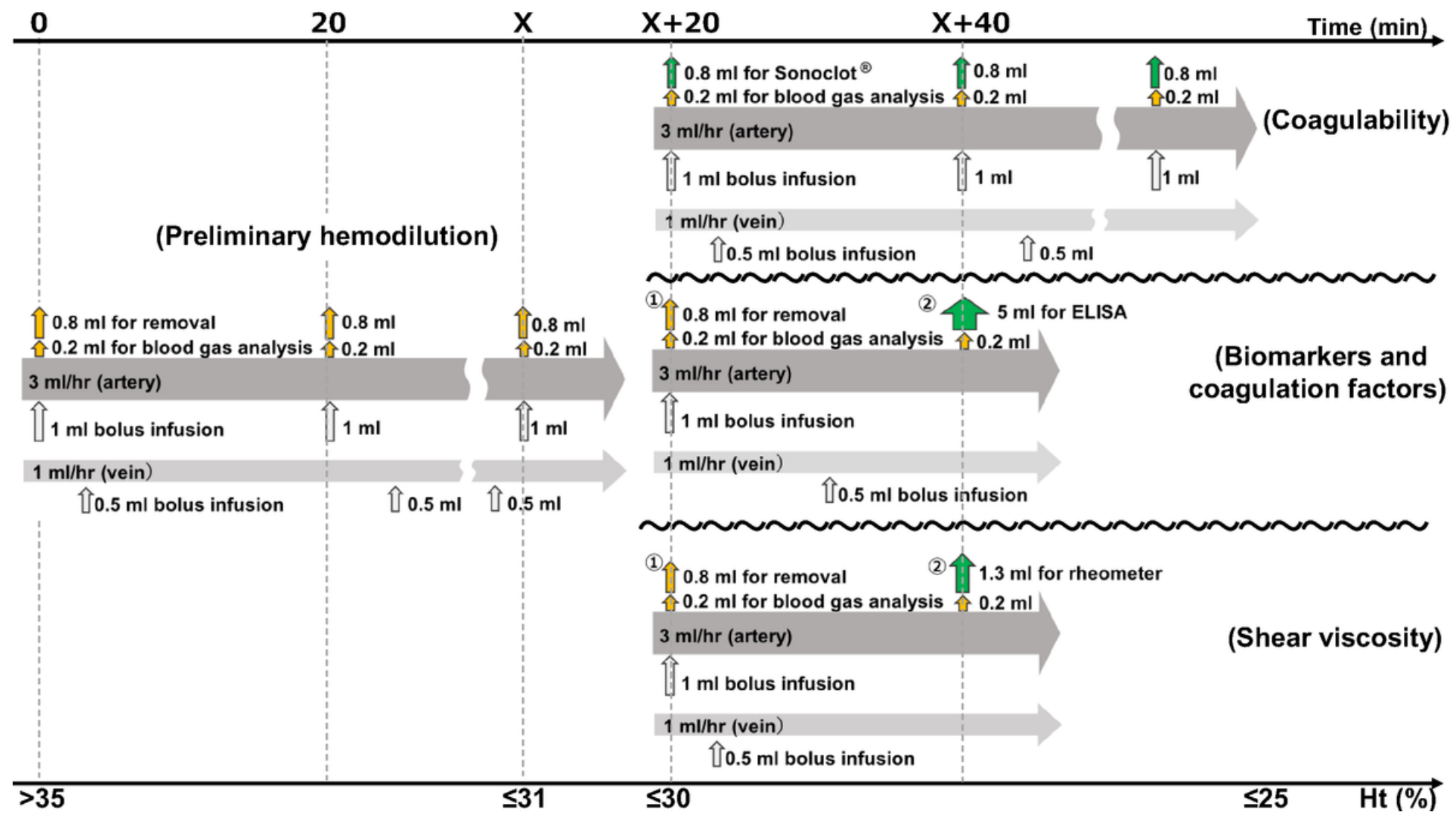

\section{Figure 1}

Hemodilution protocol. All hemodilution procedures were performed consecutively, first by the preliminary hemodilution method, followed by secondary hemodilution in different ways depending on what was measured. $\mathrm{Ht}$, hematocrit; ELISA, enzyme-linked immunosorbent assay. X, time at which the $\mathrm{Ht}$ level reached $\leq 31 \%$. Yellow arrow, external; green arrow, external (sampling); white arrow, internal 
a

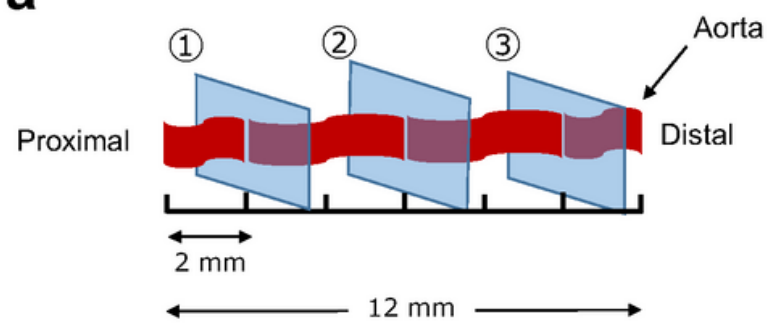

d

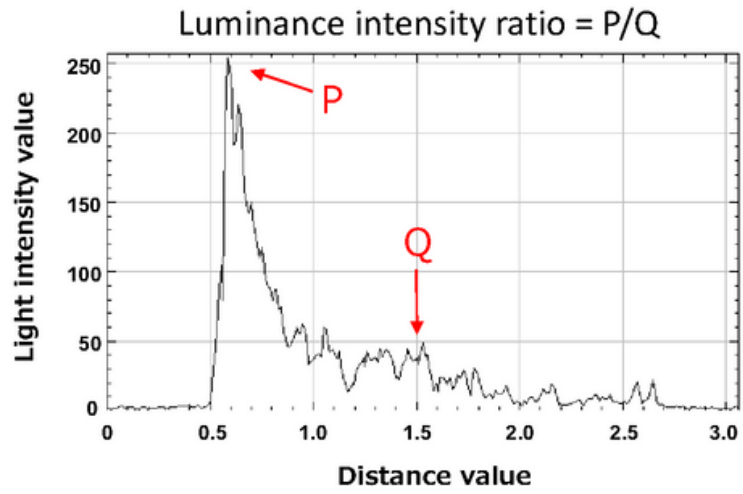

C
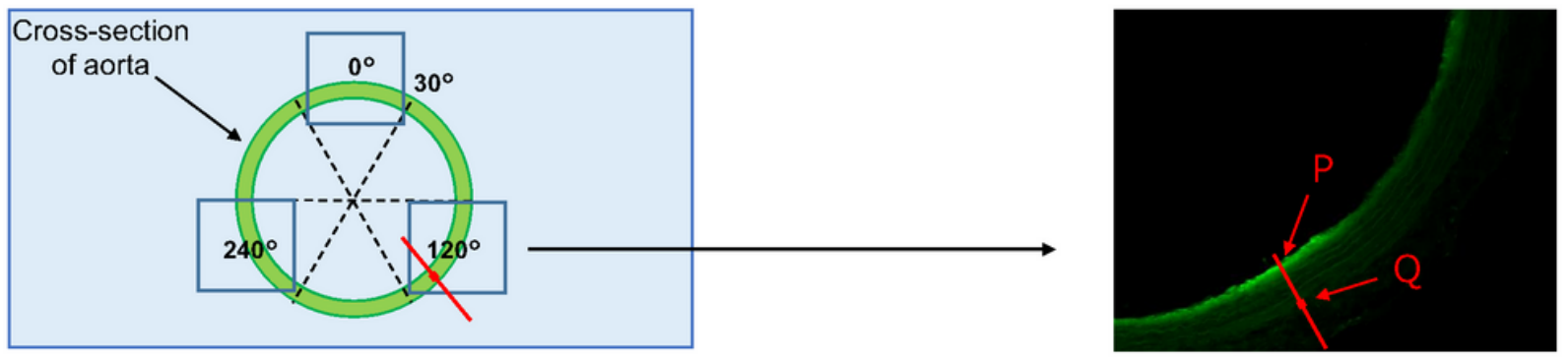

Figure 2

Evaluation of fluorescently labeled HES localized in the isolated aorta. a Collection of cross-sections from the isolated aorta. b Three images (squares) were taken in one cross-section divided into 6 parts. c A normal line was drawn from the point of highest luminance intensity on the endothelium $(P)$ to the outer membrane $(\mathrm{Q})$. $d$ Endothelial luminance intensity ratio $(\mathrm{P} / \mathrm{Q})$

A.

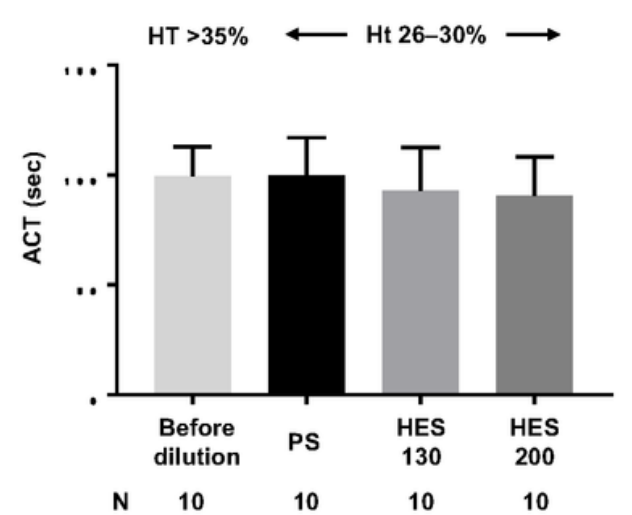

B.

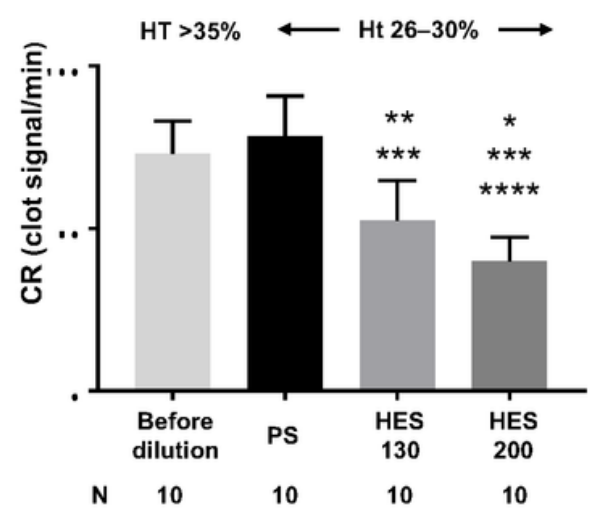

C.

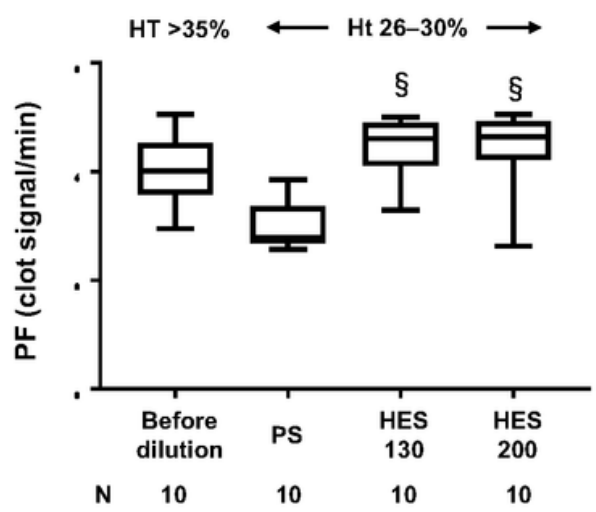

Figure 3

Coagulability measurement using Sonoclot ${ }^{\circledR}$. ACT, activated clotting time; $\mathrm{CR}$, clot rate; PF, platelet function. PS, physiological saline; HES 130, $6 \%$ hydroxyethyl starch 130/0.4 in PS (6\% Voluven); HES 200, 
10\% hydroxyethyl starch 200/0.5 in PS (10\% Pentaspan); Ht, hematocrit. *P $<0.0001$ vs before dilution,

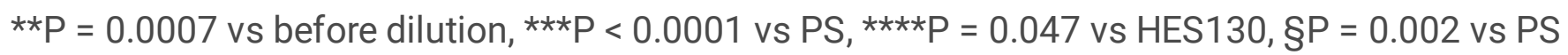

A.

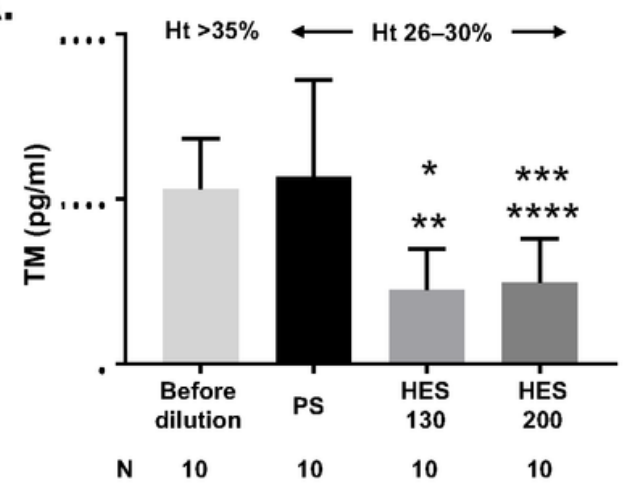

C.

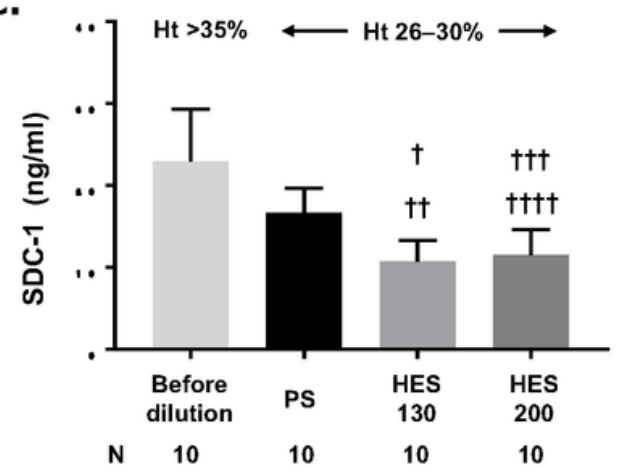

B.

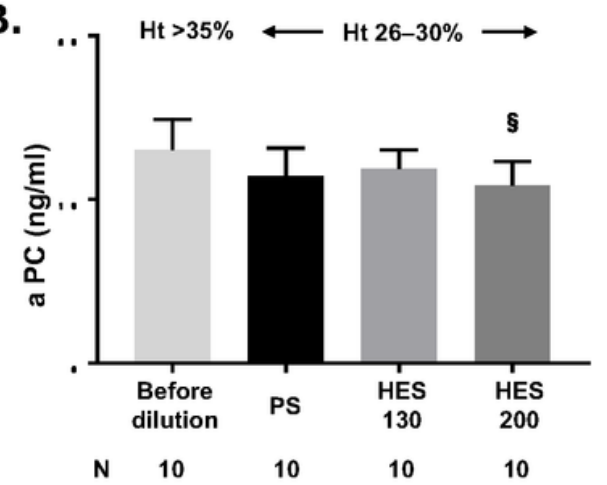

D.

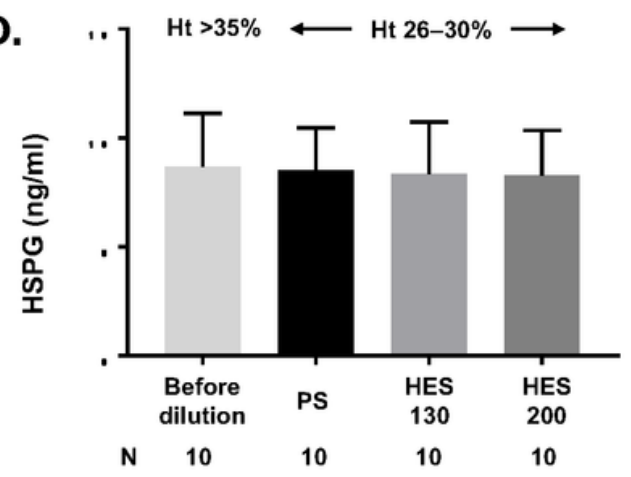

Figure 4

Quantification of ET and GCX damage markers by ELISA. ET, endothelium; GCX, glycocalyx; ELISA, enzyme-linked immunosorbent assay; TM, thrombomodulin; aPC, activated protein C; SDC-1, syndecan-1; HSPG, heparan sulfate proteoglycan. ${ }^{\star} \mathrm{P}=0.003$ vs $\mathrm{PS},{ }^{*} \mathrm{P}=0.008$ vs before dilution, $* \star * P=0.005$ vs $\mathrm{PS}$, $\star \star \star \star P=0.016$ vs before dilution, $\S \mathrm{P}=0.021$ vs before dilution, $+\mathrm{P}=0.018$ vs $\mathrm{PS},+\uparrow \mathrm{P}=0.0009$ vs before dilution, + $+\mathrm{P}=0.045$ vs $\mathrm{PS}$, †††P $=0.003$ vs before dilution

A.

$\mathrm{Ht}>35 \% \longleftarrow \mathrm{Ht} 26-30 \% \longrightarrow$

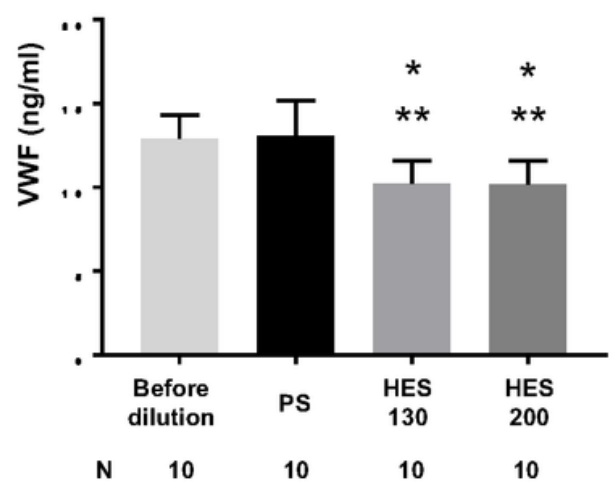

B.

$\mathrm{Ht}>35 \%$

Ht 26-30\% $\longrightarrow$

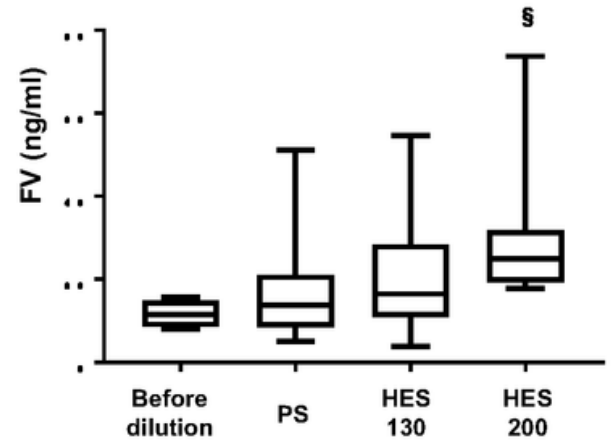

N 10
C. $\mathrm{Ht}>35 \% \longleftarrow \mathrm{Ht} 26-30 \% \longrightarrow$

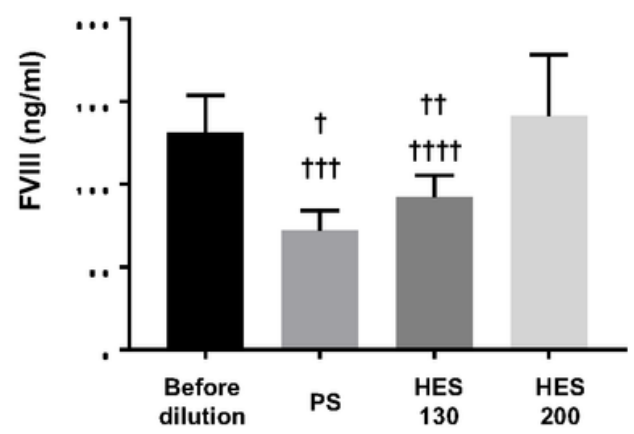

$\begin{array}{lllll}\text { N } & 10 & 10 & 10 & 10\end{array}$ 
Quantification of coagulation factors by ELISA. ELISA, enzyme-linked immunosorbent assay; VWF, von Willebrand factor; FV, coagulation factor V; FVIII, coagulation factor VIII. $* P=0.001$ vs $P S, \star * P=0.003$ vs before dilution, $\S \mathrm{P}=0.03$ vs before dilution, $\mathrm{tP}<0.0001$ vs before dilution, $\mathrm{T} \mathrm{P}=0.004$ vs before dilution, †††P $<0.0001$ vs HES200, ††††P $=0.0002$ vs HES 200
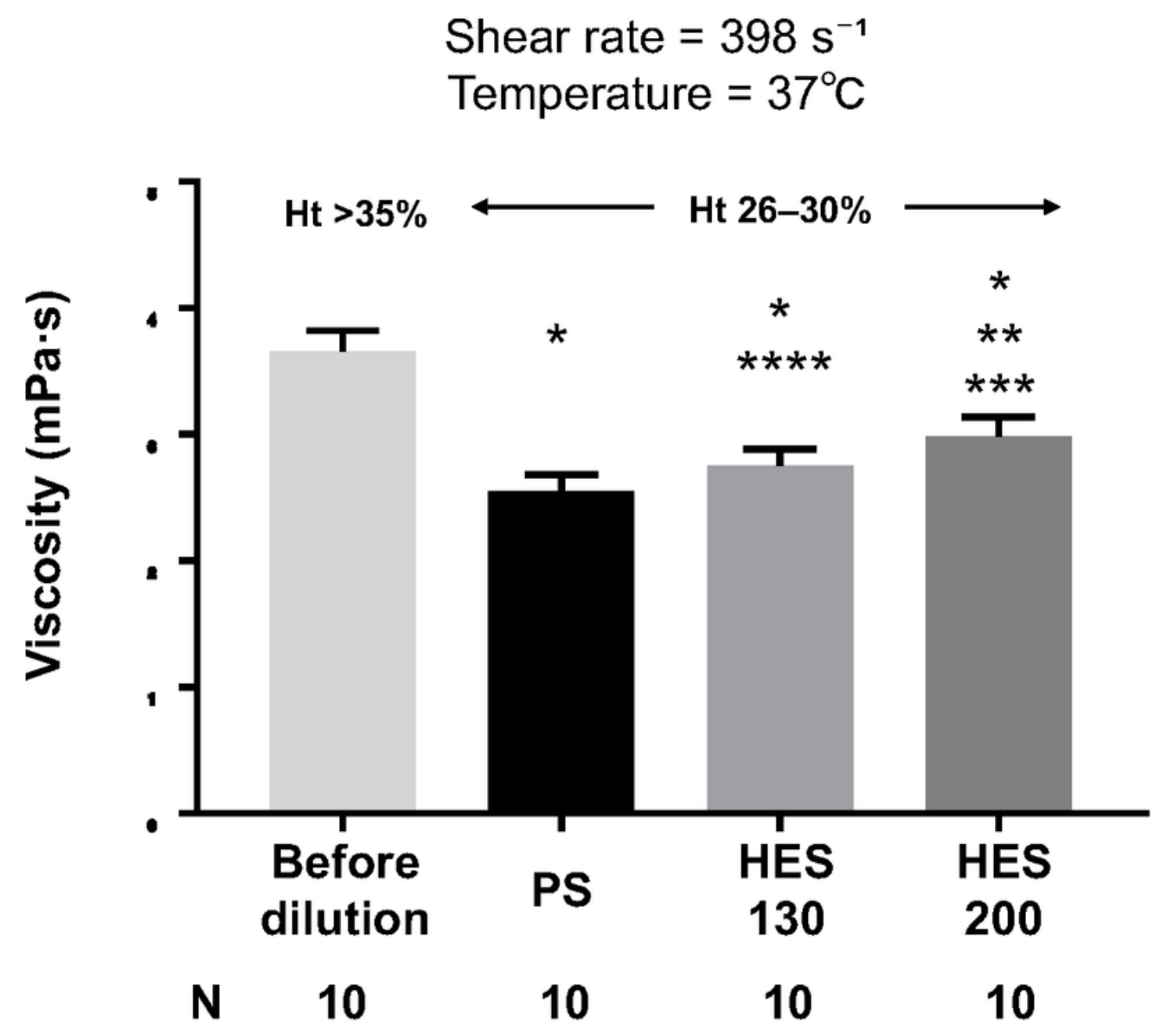

Figure 6

Shear viscosity measurement by rheometer. PS, physiological saline; HES130, $6 \%$ hydroxyethyl starch 130/0.4 in PS (6\% Voluven); HES200, 10\% hydroxyethyl starch 200/0.5 in PS (10\% Pentaspan). *P <

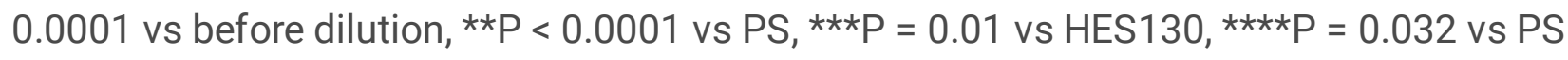



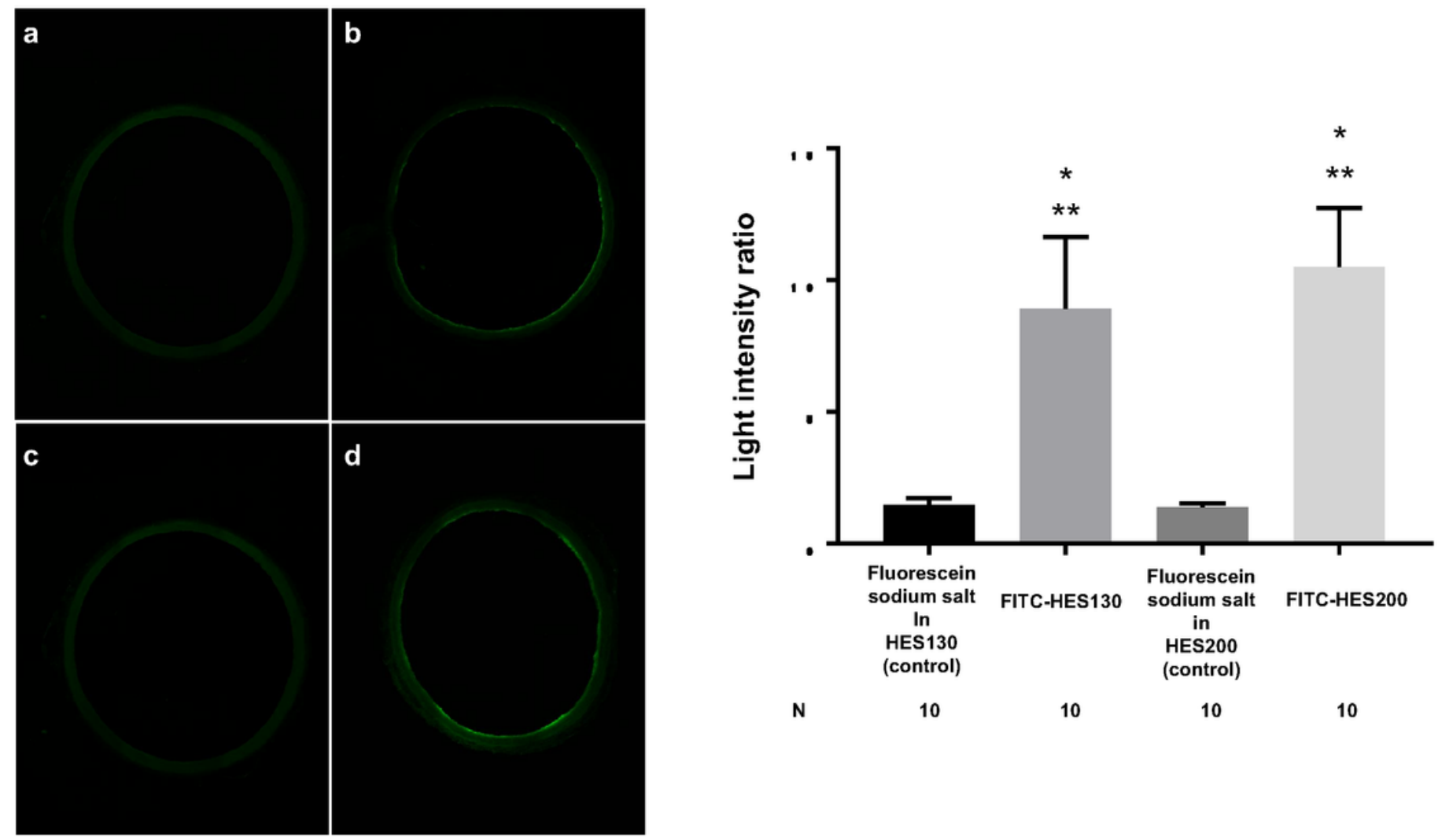

Figure 7

Left: Representative cross-sectional images of the isolated aorta after infusion of fluorescently labeled HES. a Fluorescein sodium salt in HES130 (control), b 6\% FITC-HES130 in PS, c fluorescein sodium salt in HES200 (control), d 10\% FITC-HES200 in PS. 6\% FITC-HES130 in PS, 6\% fluorescein isothiocyanate labeled hydroxyethyl starch 130/0.4 in PS; 10\% FITC-HES200 in PS, 10\% fluorescein isothiocyanate labeled hydroxyethyl starch 200/0.5 in PS. Right: Luminance intensity ratio of endothelium to outer membrane after infusion of fluorescently labeled HES in the isolated aorta. 6\% FITC-HES130 in PS, 6\% fluorescein isothiocyanate labeled hydroxyethyl starch 130/0.4 in PS; 10\% FITC-HES200 in PS, 10\% fluorescein isothiocyanate labeled hydroxyethyl starch $200 / 0.5$ in PS. ${ }^{*} P<0.0001$ vs fluorescein sodium salt in HES130 (control), **P $<0.0001$ vs fluorescein sodium salt in in HES200 (control) 